\title{
Lesions to Lateral Prefrontal Cortex Impair Lexical Interference Control in Word Production
}

\author{
Vitória Piai ${ }^{1,2 *}$, Stéphanie K. Riès ${ }^{1,2}$ and Diane Swick $k^{2,3}$ \\ ${ }^{1}$ Department of Psychology, Helen Wills Neuroscience Institute, University of California, Berkeley, Berkeley, CA, USA, \\ ${ }^{2}$ VA Northern California Health Care System, Martinez, CA, USA, ${ }^{3}$ Department of Neurology, University of California, Davis, \\ Davis, CA, USA
}

Speaking is an action that requires control, for example, to prevent interference from distracting or competing information present in the speaker's environment. Control over task performance is thought to depend on the lateral prefrontal cortex (PFC). However, the neuroimaging literature does not show a consistent relation between left PFC and interference control in word production. Here, we examined the role of left PFC in interference control in word production by testing six patients with lesions to left PFC (centered around the ventrolateral PFC) on a control-demanding task. Patients and age-matched controls named pictures presented along with distractor words, inducing within-trial interference effects. We varied the degree of competing information from distractors to increase the need for interference control. Distractors were semantically related, phonologically related, unrelated to the picture name, or neutral $(X X X)$. Both groups showed lexical interference (slower responses with unrelated than neutral distractors), reflecting naming difficulty in the presence of competing linguistic information. Relative to controls, all six left PFC patients had larger lexical interference

OPEN ACCESS

Edited by:

Joshua Oon Soo Goh, National Taiwan University, Taiwan

Reviewed by: Chris F. Westbury, University of Alberta, Canada Peter Indefrey, University of Dusseldorf, Germany

*Correspondence: Vitória Piai v.piai.research@gmail.com

Received: 8 October 2015 Accepted: 23 December 2015 Published: 20 January 2016

Citation: Piai V, Riès SK and Swick D (2016) Lesions to Lateral Prefrontal Cortex Impair Lexical Interference Control in Word Production.

Front. Hum. Neurosci. 9:721. doi: 10.3389/fnhum.2015.00721 effects. By contrast, patients did not show a consistent semantic interference effect (reflecting difficulty in selecting amongst semantic competitors) whereas the controls did. This suggests different control mechanisms may be engaged in semantic compared to lexical interference resolution in this paradigm. Finally, phonological facilitation (faster responses with phonological than unrelated distractors) was larger in patients than in controls. These findings suggest that the lateral PFC is a necessary structure in providing control over lexical interference in word production, possibly through an early attentional blocking mechanism. By contrast, the left PFC does not seem critical in semantic interference resolution in the picture-word interference paradigm.

Keywords: Bayesian estimation, Broca's area, competition, cognitive control, lexical selection, selective inhibition

\section{INTRODUCTION}

Speaking is a seemingly effortless task executed on a regular basis. Yet, speaking is a controlled action (e.g., Roelofs and Piai, 2011). As a broad term, control refers to regulatory/monitoring processes that ensure that our actions are in agreement with our goals, especially in the face of distraction (e.g., Posner and Petersen, 1990). We need to exert control over language production for various reasons. For example, speakers need to prevent interference from concurrent visual or auditory information present in their environment. Moreover, when retrieving 
words from long-term memory, associated information that is not relevant to the task at hand is also retrieved. Control must also be exerted over these interfering memory representations so the appropriate word can be selected (e.g., Badre and Wagner, 2007). Whereas the neuroanatomical characterization of core language-production processes has advanced considerably (e.g., Indefrey, 2011; Price, 2012), only recently has research been dedicated to the control aspects of production (e.g., Alario et al., 2006; Piai et al., 2013; Geranmayeh et al., 2014; Riès et al., 2014, 2015). In the present study, we examined the role of left lateral prefrontal cortex (PFC) in control over language production by testing stroke survivors with damage to left PFC on a control-demanding task. We varied the type of interfering information in order to increase the difficulty of picture naming, as we explain next.

A fruitful paradigm to investigate control functions implemented during speaking is picture-word interference (Rosinski et al., 1975). In this task, speakers are instructed to name pictures as fast and accurately as possible, while ignoring a distractor word superimposed on the picture. The distractor word can be manipulated to provide information (partially) congruent with the picture name (e.g., the picture of a carrot with the distractor "castle", which overlap in their initial phonemes) or incongruent (e.g., pictured carrot, distractor "stamp"). Control functions are assumed to aid the production of the picture name in the presence of incongruent, competing information from the distractor word. In particular, naming pictures in the presence of distractor words likely requires interference control, an executive function involved in "suppressing a stimulus that pulls for a competing response so as to carry out a primary response, [...] suppressing distractors that might slow the primary response [...]" (Nigg, 2000, p. 222; for further discussion, see also Friedman and Miyake, 2004).

The type of relationship that the picture name bears with the distractor is an important determinant of people's performance. For example, naming a picture with an unrelated distractor word (e.g., the picture of a carrot with the distractor "stamp") is slower than naming a picture with a series of Xs superimposed (e.g., Glaser and Düngelhoff, 1984). In the neutral condition (XXX), only the picture name is activated by the picture stimulus, whereas with unrelated distractor words, both distractor word and picture name activate their representations, which then compete during word planning. We refer to this effect as the lexical interference effect. Furthermore, if the picture and distractor are from the same semantic category (e.g., pictured carrot, distractor: "radish"), picture naming is slowed down relative to an unrelated distractor. This effect is known as the semantic interference effect (e.g., Glaser and Düngelhoff, 1984). In this case, the semantic relation between picture and distractor makes the distractor a stronger competitor for the picture name relative to an unrelated word (e.g., Roelofs, 1992, 2003). By contrast, if the picture and the distractor share a phonological relationship (e.g., pictured carrot, distractor: "castle"), picture naming is faster relative to an unrelated distractor. This effect is known as the phonological facilitation effect (e.g., Schriefers et al., 1990). The phonological overlapping information between the distractor and the picture name preactivates the picture name, facilitating its production.

Importantly, lexical and semantic interference possibly originate at different processing stages in language production. In particular, the semantic interference effect has been attributed to selection difficulty arising at the lexical level (e.g., Roelofs, 1992). Differently, the lexical interference effect could potentially reflect the operation of an early attentional blocking mechanism, which is more challenged in the presence of distractor words compared to the neutral condition (XXX; e.g., Roelofs, 2003; Roelofs et al., 2011). Whether the same brain region underlies the control mechanisms engaged in resolving these interference effects is unclear.

\section{Neuroanatomical Findings}

The lateral PFC is known to be involved in broad aspects of topdown control over task performance (e.g., Petrides, 2005), such as monitoring and manipulating information, and regulating selection amongst competing representations (for reviews and meta-analyses, see e.g., Alvarez and Emory, 2006; Szczepanski and Knight, 2014; Yuan and Raz, 2014). With respect to pictureword interference, a number of neuroimaging studies have used different types of distractor words. However, no studies have examined lexical interference, and the evidence for differential activation in the PFC for semantic interference is mixed. Using auditory distractor words, one study found that the semantic interference effect was associated with modulations in the ventrolateral PFC activation (de Zubicaray and McMahon, 2009). In the other four studies, however, differential activation to distractors was observed in brain areas other than the lateral PFC, including the anterior cingulate and different structures within the left temporal cortex. For example, two studies found no increases in brain activity for the semantically related relative to the unrelated condition, only activity decreases in left temporal cortex (de Zubicaray et al., 2013; Piai et al., 2013). One of these studies also found activity increases in the anterior cingulate cortex when comparing semantically related and identity distractors (i.e., Piai et al., 2013). Finally, one study compared semantically related distractors to a neutral condition (XXX), which is an unusual comparison, and found anterior cingulate and superior frontal gyrus involvement (de Zubicaray et al., 2001). The involvement of lateral PFC in resolving the lexical interference effect therefore remains an open question.

Functional imaging studies can inform us about the engagement of brain areas in a particular function. However, claims regarding the necessity of those areas for a particular function are mainly possible by means of lesion-symptom investigations. Previous neuropsychological studies have primarily focused on the semantic interference and phonological facilitation effects. For example, a picture-word interference study conducted on aphasic individuals found increased semantic interference and increased phonological facilitation effects in aphasics relative to age-matched controls (Hashimoto and Thompson, 2010). However, lesion location for these 
patients was not provided. Biegler et al. (2008) also observed an increased semantic interference effect in two of their patients with left frontal lesions, whereas the third patient, with unknown lesion location, showed no semantic interference effect. To the best of our knowledge, there is no published picture-word interference study on a group of patients with well-characterized, focal PFC lesions. Furthermore, no neuropsychological study has examined the lexical interference effect (for lesion studies on other picture-naming tasks, see Schnur et al., 2006; Riès et al., 2014, 2015).

This brief review of the neuroimaging literature does not show a consistent relation between left PFC and the resolution of distractor interference in picture naming. Moreover, the current neuropsychological literature does not provide enough anatomical specification associated with impairments in interference control in picture naming. Finally, since the studies discussed above assessed the effect of competing semantic alternatives only, it remains unclear whether the left PFC is necessary in providing control over lexical interference in word production. Specifically, does the left PFC support control over word production processes in the presence of any distracting or competing word? Control over word production amidst competing (unrelated) alternatives would be indicated by increased interference from distractor words, which provide competing linguistic information, relative to a neutral condition (XXX), in which no competing linguistic information is present. If the left PFC is necessary for lexical interference control in word production, then the lexical interference effect should be larger in PFC patients relative to controls.

\section{The Present Study}

We employed the picture-word interference task to examine the role of left PFC in interference control over word production. We tested six chronic stroke patients with damage to the left PFC and 13 age-matched controls on their picturenaming performance while varying the difficulty of word production by having semantically related, phonologically related, unrelated, and neutral (XXX) distractors. We assessed (1) whether patients and controls differed in overall performance; (2) whether distractor effects were present in the overall data and most importantly; and (3) whether the magnitude of each of these effects was comparable between patients and controls. Since we were interested in interference effects, we opted for maximizing the interference of distractors by presenting the picture and the distractor simultaneously (as is classically done in the picture-word interference task; e.g., Damian and Martin, 1999). Therefore, no manipulation of stimulus onset asynchrony was introduced.

The response times (RTs) were analyzed within a robust Bayesian estimation framework. We chose this type of analysis over more traditional null hypothesis significance testing for a number of reasons. Firstly, with this approach, we can provide richer information (i.e., an explicit posterior distribution) on how groups differ in the conditions under analysis and a more reliable estimate of this difference. Secondly, with an analysis of variance (ANOVA) approach, a multiple comparisons problem may exist (cf. Cramer et al., 2015). With the Bayesian approach adopted here, all parameters are estimated simultaneously, creating one fixed posterior distribution from which all effects are investigated, obviating the multiple comparisons problem. Finally, using Bayesian statistics, one can directly test whether there are no statistical differences between conditions. In null hypothesis significance testing, one could only reject the null hypothesis of no differences between patients and controls. However, one could not accept the null hypothesis that patients and controls do not differ in their behavior. Within a Bayesian framework, one can potentially also accept the null value (i.e., no difference between conditions compared) when the certainty in the estimate is high.

\section{MATERIALS AND METHODS}

The study protocol was approved by the Institutional Review Board of the Northern California Health Care System, following the Declaration of Helsinki. Participants gave written informed consent and were paid for taking part in the study.

\section{Participants}

Six patients (five males, mean age $=66$ years, mean education $=14$ ) with focal damage to the left lateral frontal cortex participated. All were chronic stroke patients tested at least 1 year post stroke. Information on their handedness as well as on their language ability is shown in Table 1. All lesions were due to infarction in the precentral branch of the middle cerebral artery. The damage was centered on the pars triangularis of the ventrolateral PFC (five of the six patients), as shown in the lesion overlap map in Figure 1. Lesions were transcribed from CT or MRI scans onto corresponding axial templates by a neurologist for reconstruction. Patients' performance on the Western Aphasia Battery (WAB; Kertesz, 1982), shown in Table 1 (for five of the six patients), indicated good language abilities in all five

\begin{tabular}{|c|c|c|c|c|c|c|}
\hline Patient & Handedness & Naming & Reading & $\mathbf{A Q}$ & MPO & Lesion vol (cc) \\
\hline P1 & Right & 60 & 100 & 98.6 & 20 & 52 \\
\hline P2 & Right & 60 & 86 & 97.9 & 55 & 37 \\
\hline P3 & Right & 60 & $N A^{*}$ & 99 & 43 & 20 \\
\hline P4 & Right & 60 & 81 & 94.7 & 53 & 54 \\
\hline P5 & Right & 60 & 84 & 96.3 & 67 & 75 \\
\hline P6 & Left & $\mathrm{NA}^{* * * *}$ & $N A^{* * * *}$ & $N A^{* * *}$ & $N A^{* *}$ & $N A^{* *}$ \\
\hline
\end{tabular}

Note: Naming (maximum score $=60$ ) and Reading (maximum score $=100$ ) scores, as well as $A Q$ (maximum score $=100$ ), are measures from the Western Aphasia Battery (WAB). *P3 was not assessed on the WAB reading tests. However, the patient scored $100 \%$ in the reading assessment of sentences and paragraphs of the Boston Diagnostic Aphasia Examination. ${ }^{* *}$ Data not available. ${ }^{* * *} P 6$ was not assessed on language batteries as he was not aphasic. AQ, Aphasia Quotient; cc, cubic centimeter; MPO, months post stroke onset; vol, volume. 


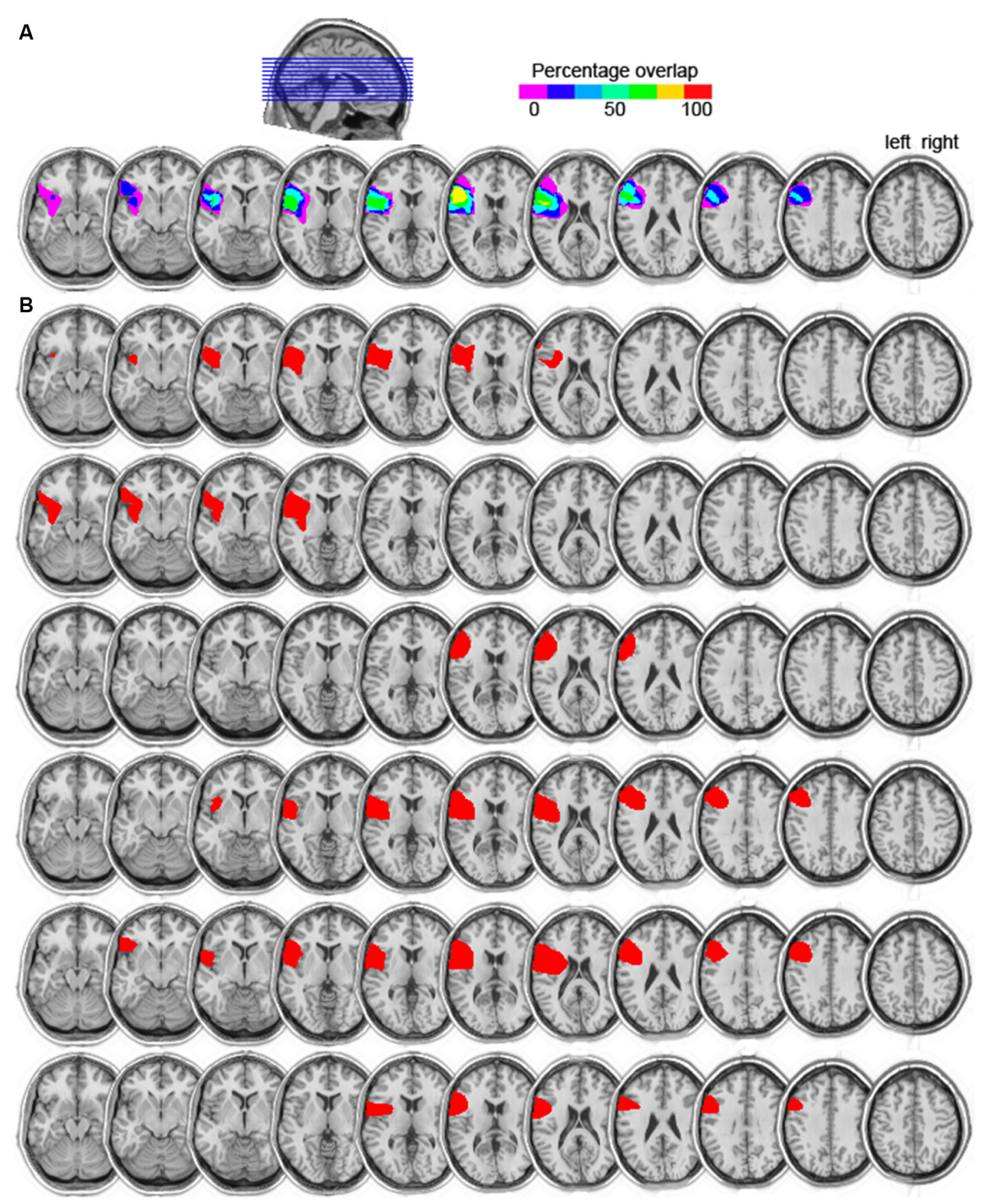

FIGURE 1 | (A) Lesion overlap map of the six left prefrontal cortex (PFC) patients. The color scale indicates the amount of overlap in lesion locations, with magenta indicating that only one patient had a lesion in that particular region (i.e., 0\% overlap). The maximum overlap, shown in yellow, indicates that five of the six patients had a lesion in that particular region (slices 6 and 7 from left to right). (B) Individual lesion reconstructions, shown in red. Each patient is shown in a subsequent row, from P1 (top) to P6 (bottom).

patients: An Aphasia Quotient (AQ) score of 93.8 or higher (out of 100) indicates performance within normal limits (Kertesz, 1982). Thus, our patients were not classified as aphasic. Additionally, 13 controls participated (five males; mean age $=66$, $S D=7$; mean years of education $=14, S D=2.5)$. They were matched to the PFC patients for age $\left(t_{(12)}<1, p=0.802\right)$, years of education $\left(t_{(8)}<1, p=0.967\right.$ ), and handedness (one left handed participant in each group). None of the patients or control participants had a history of psychiatric disturbances, substance abuse, medical complications, multiple neurological events, or dementia.

\section{Stimuli}

Twenty-two pictures of objects were selected from Snodgrass and Vanderwart (1980). Each object was an exemplar of a different category. Three different types of distractor word were selected for each picture (materials were adapted from Taylor and Burke, 2002). In the related condition, distractors were taken from the same taxonomic category as the picture (e.g., picture: carrot, distractor: "radish"). In the phonological condition, a phonologically related (but semantically unrelated) word was chosen with the same two initial phonemes as the picture name (e.g., picture: carrot, distractor: "castle"). 
In the unrelated condition, the distractor was a semantically and phonologically unrelated word (e.g., picture: carrot, distractor: "stamp"). Finally, in the neutral condition, the picture was presented with XXX superimposed. Each distractor word appeared only once in the experiment. Distractors were matched for length (number of letters) and for frequency $\left(F^{\prime} s_{(1,64)}<1\right)^{1}$. Pictures were presented once with each distractor, yielding a total of 88 experimental trials.

\section{Procedure}

First, the participants were shown sheets of paper containing the pictures that were to appear in the practice and experimental trials, and were asked to name each one. If an incorrect response was given, the experimenter presented the correct name. Once this procedure was completed twice, testing on the computer began. Stimulus presentation and response recording were controlled by the Presentation Software (Neurobehavioral Systems, Albany, CA, USA). Each participant was seated in front of the monitor while wearing a headset with a microphone. The instructions informed participants that they would be naming pictures appearing on the screen and that, in addition, a word or XXX would be superimposed on each picture. They were instructed to ignore the distractor and name the picture on the screen as quickly as possible, avoiding errors or extraneous vocalizations. To begin each trial, the word "Ready" appeared on the monitor for $1500 \mathrm{~ms}$. Next, a picture appeared simultaneously with a distractor stimulus and remained on the screen until a spoken response was made or $5000 \mathrm{~ms}$ had passed, and then the stimuli disappeared. The screen remained blank for $1000 \mathrm{~ms}$ before the following sentence appeared: "The correct answer is BLANK [the correct name of the object]. If you said BLANK, press the space bar." This sentence appeared on the screen until the participant pressed the space bar or $3000 \mathrm{~ms}$ had passed. This self-evaluation procedure served unrelated purposes and these responses were not analyzed here. Note, however, that this procedure was presented for every participant and condition and as such, is not confounded with our distractor manipulation nor with participant group. The next trial followed $1000 \mathrm{~ms}$ later. There were seven practice trials followed by 88 experimental trials. RTs were measured by means of a voice key included in the Presentation software. The voice key was calibrated for each participant during the practice.

\section{Analysis}

All data analysis was conducted using R ( R Development Core Team, 2014) and "rjags" (Plummer, 2014). The experimenter monitored naming responses online. All trials with disfluent or incorrect responses were coded as errors and subsequently

\footnotetext{
${ }^{1}$ Unintentionally, one unrelated and two phonological distractors chosen were adjectives rather than nouns ("tactic", "antique", "frozen"), and one distractor was not a concrete noun ("dream"). We note that the pattern of results was the same whether we included or excluded these items, so the results reported comprise all distractors.
}

excluded from the RT analysis. Errors comprised between 0 and $3.8 \%$ of the patients' responses and between 0 and $2.8 \%$ for the controls, with no significant differences between patients and controls for any of the distractor conditions (logistic regression, $p$ 's $>0.09$ ). Trials with voice-key triggering failure $(5.3 \%$ of the total number of trials for patients and $3 \%$ for controls) or RTs shorter than $200 \mathrm{~ms}$ (one trial for one patient) were further excluded from the RT analysis. The RT data of both control and patient groups were not normally distributed. Given that the median is the best representative of central tendency with skewed data, participants' median RTs were computed for each condition.

The RTs were analyzed with a hierarchical Bayesian estimation approach (Kruschke, 2015, available at http://www. indiana.edu/ kruschke/DoingBayesianDataAnalysis/Programs/ SplitPlotJags.R). Under this approach, the data are modeled mathematically and belief is reallocated away from parameter values that are not consistent with the data in favor of parameter values consistent with the data. As such, inference from data does not rely on a $p$ value. We used the $95 \%$ highest density interval (HDI) of the posterior distribution to decide whether to reject or accept the null value. The HDI summarizes a belief distribution such that values inside the HDI have a higher probability than values outside the HDI. If the HDI includes zero, then the difference between the estimated parameters describing the data in each condition is credibly zero. Thus, in this case, there is evidence in favor of the hypothesis of no differences between conditions.

The model we used can be related to an ANOVA in that we can examine the between-participant factor group (controls vs. patients), the within-participant factor distractor effect (lexical and semantic interference, and phonological facilitation) for each group, and their interaction (i.e., whether the magnitude of the distractor effects differ between patients and controls).

The data were modeled as coming from a $t$ distribution. Each group by distractor combination is described with a $t$ distribution with its own mean. The parameters describing the standard deviation $(S D)$ and the tails of the distribution (i.e., the nu parameter) were the same for all group by distractor combinations so that their estimation is informed by data from both groups and all distractor conditions (e.g., Kruschke, 2013). The nu parameter represents the degrees of freedom of the $t$ distribution, with small values indicating heavy tails and large values indicating a nearly normal distribution. The effect of each level of a factor on the data is described as a deflection away from the overall central tendency (baseline) of the data. These deflections respect the constraint that they must sum to zero. The variance of the deflections (i.e., the precision) is then estimated from the data (Kruschke, 2015). Whereas in the ANOVA framework, different comparisons rely on different error terms for computing the $F$ ratio, in this Bayesian ANOVA, the parameters of all deflections are estimated simultaneously, creating one fixed posterior distribution. This posterior distribution is then used to examine the main, interaction, and simple effects of interest, obviating the multiple comparisons problem. 
In this implementation, we have chosen prior distributions that are noncommittal and vague, having virtually no influence on the parameter estimates (Kruschke, 2013, 2015). The baseline value had a normal prior with a mean of 908 (the mean of the pooled data) and its precision was $1.0 \mathrm{e}-6$ times the precision of the pooled data (i.e., 1.0e-11). The prior for the $S D$ had a uniform distribution, with the lowest and highest values considered being the $S D$ of the pooled data divided or multiplied by 1000 , respectively. The prior on the nu parameter, describing the tails of the distribution, had a prior with an exponential distribution $($ mean $=29)$. This value is optimal for balancing between nearly normal and heavy-tailed distributions. This distribution assigns equal credibility to nearly normal and heavily tailed data (Kruschke, 2013). The priors for the effects (deflections from baseline) were also noncommittal, described with a normal distribution with a mean of zero. Its precision parameter had a gamma prior parameterized by the mean $(5.0 \mathrm{e}-05)$ and $1.0 \mathrm{e}+6$ times the $S D$ (33) of the observed precisions of all group by distractor combinations, yielding shape $=2 \mathrm{e}-12$ and rate $=4.6 \mathrm{e}-8$. Importantly, choices of different priors and prior distributions did not affect the pattern of results obtained. The posterior distribution was approximated using a Markov chain Monte Carlo method with 100,000 samples. The chains were initialized based on the data, that is, the mean and $S D$ of the pooled data, as well as of the factors group and distractor. In order to avoid problems with highly autocorrelated chains, the chains were thinned such that only each 50th step in the chain was used. The burn-in period was set to 2000 steps, that is, the first 2000 steps of the chain were discarded as they are not as well representative of the data as the later steps are. For completeness, we report the results of repeated measures ANOVA (uncorrected for multiple comparisons, cf. Cramer et al., 2015), with distractor type (related, unrelated, phonological, neutral) as a within-participants variable and group (patients, controls) as a between-participants variable.

\section{RESULTS}

The semantic interference and phonological facilitation effects were calculated relative to the unrelated condition (i.e., semantic interference: related-unrelated; phonological facilitation: phonological-unrelated), whereas the lexical interference effect was calculated as unrelated-XXX. In this way, interference is indicated by positive values and facilitation by negative values.

Figure 2 (left) shows a box-and-whisker plot of the participants' median RTs. The right panel of Figure 2 shows how many $S D$ s each patient's performance is relative to the mean of the control participants for each effect of interest. Dashed lines indicate $2 \mathrm{SDs}$. It is clear from the figure that, for the lexical interference effect, all patients scored more than $2 S D$ s above the mean of the controls, suggesting relative impaired performance consistent across all patients. For the semantic interference effect, however, only two patients scored beyond $2 S D$ s, one above the mean and one below the mean of the control participants. Finally, for the phonological facilitation effect, five of the six patients scored more than $2 S D$ s below the mean of the control participants, suggesting that phonological distractors convey an additional benefit for the patients in picture naming relative to controls.

The results of the Bayesian estimation are summarized in Figures 3, 4. For each effect, a histogram is shown of 100,000 credible parameter values from the posterior distribution, given the data. The mean of the parameter values for each distribution is indicated on top. The $95 \% \mathrm{HDI}$ is indicated for each histogram as a thick horizontal line below the distribution.

For the group effect (patients vs. controls), shown in Figure 3, the $95 \% \mathrm{HDI}$ is clearly greater than zero (range: $337-438$ ) and in fact, $100 \%$ of the credible parameter values of the difference in their performance is greater than zero. Thus, patients' overall picture naming performance is credibly slower than in controls.
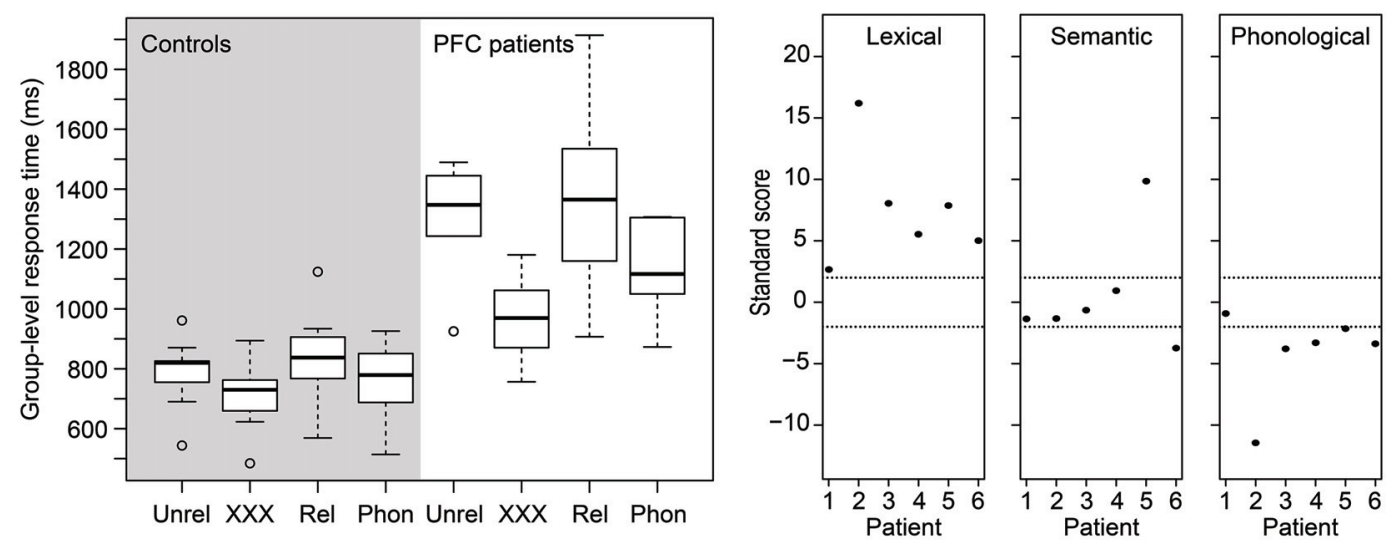

FIGURE 2 | (Left) Box-and-whisker plot of the picture naming times of the age-matched controls (left panel) and of the patients (right panel). (Right) Standardized scores of the patients' distractor effects. Dashed horizontal lines indicate 2 SDs (approximately the 95th percentile). PFC, prefrontal cortex; Phon, phonological; Rel, semantically related; Unrel, unrelated. 


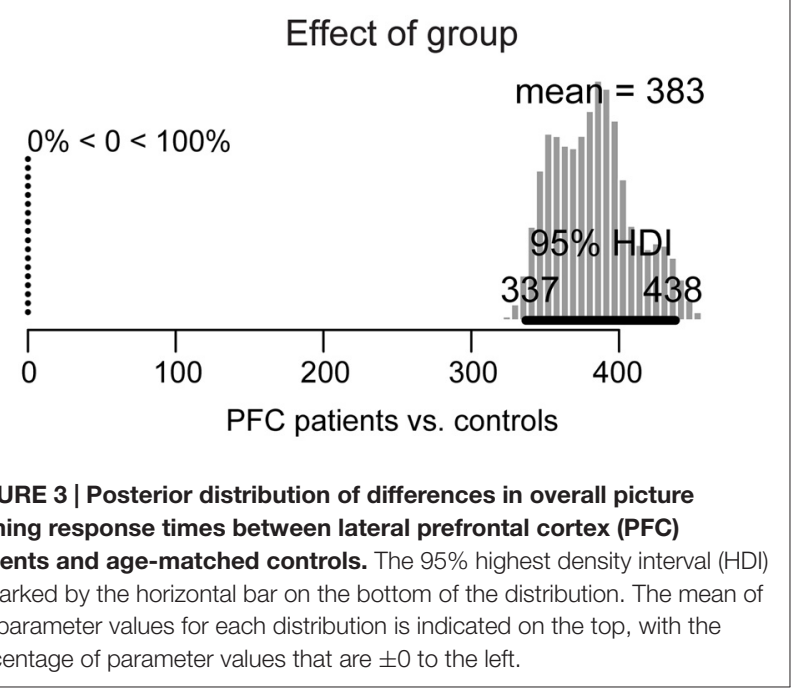

The next comparisons of interest are the distractor effects, shown in Figure 4. Interference effects are represented by positive values whereas facilitation effects by negative values.

The distractor effects in the control group are shown in the top row of Figure 4. For the lexical interference effect (unrelated vs. XXX, left panel), the 95\% HDI clearly does not include zero (range: 57-100), with $100 \%$ of the credible parameter values greater than zero. Similarly, for the semantic interference effect (related vs. unrelated, middle panel), the $95 \%$ HDI is greater than zero (range: $12-57$ ), with $100 \%$ of the credible parameter values greater than zero. Finally, for the phonological facilitation effect (phonological vs. unrelated, right panel), the 95\% HDI does not include zero (range: -48 to -3 ), with $98 \%$ of the credible values smaller than zero. Thus, the lexical interference, semantic interference, and phonological facilitation effects are all credibly present in the data.

The distractor effects of the PFC patients are shown in the middle row of Figure 4. For the lexical interference effect (left panel), the 95\% HDI clearly does not include zero (range: 227-349), with $100 \%$ of the credible parameter values greater than zero. By contrast, the semantic interference effect (middle panel) is not credibly present in the data: the 95\% HDI includes zero (range: -143 to 108) with the credible parameter values almost symmetrically distributed below and above zero. Finally, for the phonological facilitation effect (right panel), the 95\% HDI clearly does not include zero (range: -178 to -83 ), with $100 \%$ of the credible values smaller than zero. In summary, whereas the age-matched controls credibly showed all three classical distractor effects (lexical and semantic interference, and phonological facilitation), the PFC patients only showed credible lexical interference and phonological facilitation.

The comparisons of effect magnitude between patients and controls are shown in the bottom row of Figure 4. Decreased performance (i.e., larger interference and smaller facilitation effects) in patients relative to controls are represented by values smaller than zero. Increased performance (i.e., smaller interference and larger facilitation effects) are represented by values greater than zero. The lexical interference effect (left panel) is credibly larger in the patient group than in controls, with a $95 \%$ HDI between -273 and -143 . In fact, $100 \%$ of the credible parameter values are smaller than zero. By contrast, the semantic interference effect is not credibly larger in patients than in controls, with the 95\% HDI including zero (range: -77 to 178). Finally, patients have a credibly larger phonological facilitation effect than controls, with the 95\% HDI between 52 and 157, and $100 \%$ of the credible values greater than zero.

The results of the ANOVA are presented in Table 2. These results largely converge with the results of the Bayesian analysis reported above. The only difference is the phonological facilitation effect in the controls, which was not significant in the ANOVA, but credible in the Bayesian analysis. However, with the Bayesian estimation we provide not only information on whether an effect is credible or not, but also a range of values for these effects that are most credible given the data.

\section{The Effect of General Slowing}

In order to assess whether the larger effect sizes in the patients than in the controls could be accounted for by general slowing, we conducted the following analysis. For each participant, we determined the RT ratio for each effect by calculating the RT difference between two conditions (lexical effect: unrelated minus neutral, semantic effect: related minus unrelated, phonological effect: phonological minus unrelated) and then dividing the difference by the RTs in the unrelated condition, which is common to all three distractor effects.

Figure 5 shows a box-and-whisker plot of the RT ratios for age-matched controls (gray-shaded panels) and patients (white-shaded panels) for the three effects. The box-and-whisker plots show little overlap in the RT ratios for the lexical and phonological effects, indicating that these two effects cannot be explained by general slowing alone. That is, the lexical interference effect in patients is still larger than in the controls after correcting for general slowing. Similarly, the phonological facilitation effect in patients is still larger than in controls after accounting for the effect of general slowing. However, for the semantic interference effect, the RT ratios indicate similar magnitude in patients and controls. These results are compatible with a lack of a difference in semantic interference effect between patients and controls, as reported above.

\section{DISCUSSION}

This study is the first to examine picture-word interference in a group of patients with well-characterized left lateral PFC lesions, maximally overlapping in the ventrolateral PFC.

Patients were overall slower in picture naming than controls (for a similar finding in aphasic patients, see e.g., Biegler et al., 2008; Hashimoto and Thompson, 2010). Importantly, picture naming with a distractor word caused more interference for patients than for controls relative to a non-linguistic distractor (XXX). Furthermore, patients showed more phonological facilitation than controls. Finally, on a group level, patients failed 


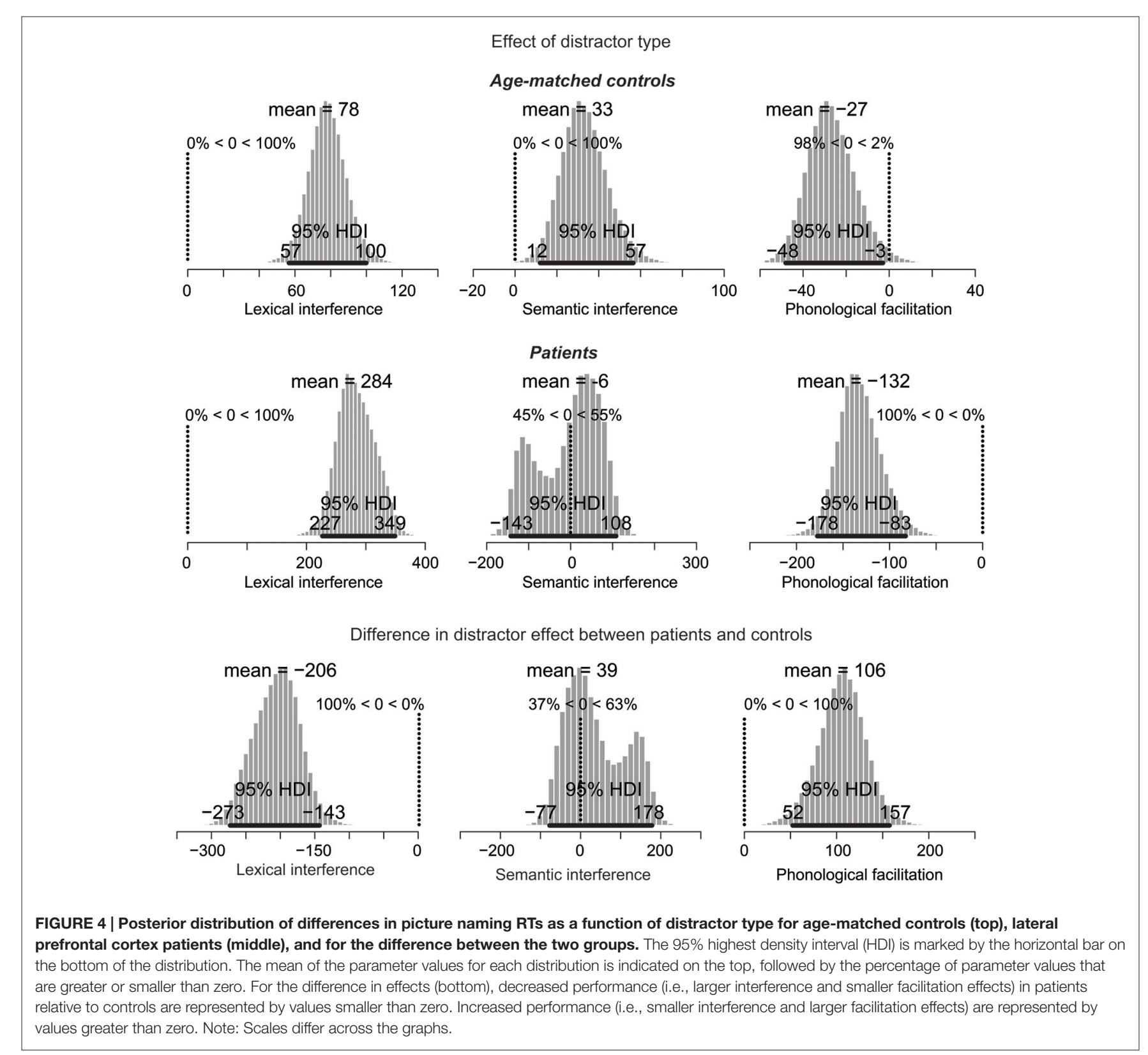

to show a reliable semantic interference effect. These findings were evident from both the Bayesian estimation results and from the standardized scores of the effects for each patient. Below, we discuss each of these effects in more detail.

A novel finding from the present study was the increased lexical interference effect for patients relative to controls. In fact, all six patients were consistently impaired by distractor words relative to neutral, non-linguistic distractors. It has been argued that interference control is a significant cognitive construct, involved in multiple tasks and related to other cognitive abilities (Unsworth, 2010). However, its functional neuroanatomy in relation to word production has remained unclear. Previous neuropsychological studies using verbal tasks have often used the color-word Stroop task (e.g., Tsuchida and Fellows, 2013;
Geddes et al., 2014), in which participants name the ink color of color names (e.g., red printed in red ink or blue printed in red ink). However, this task requires multiple repetitions of a few words (typically, 3-5 color names) and as such, is not ideal for investigating word production processes. Evidence that word planning depends on attentional control is now substantial (for review, see Roelofs and Piai, 2011) and recently, it has also been shown that interference control (also termed selective inhibition) plays an important role in picture-word interference (e.g., Shao et al., 2015). Our finding that competing linguistic information is especially problematic for all our patients with lateral PFC damage not only strengthens the previous relation between interference control and word production, but also further specifies that this type of control necessarily implicates the 
TABLE 2 | Results of the repeated measures analysis of variance.

\begin{tabular}{|c|c|c|c|c|c|c|}
\hline & Mean diff (ms) & $\boldsymbol{F}$ & $t$ & $d f$ & $P$ & $95 \% \mathrm{Cl}$ \\
\hline \multicolumn{7}{|l|}{ Effect } \\
\hline Main: patient vs. control & 415 & 34.97 & & 1,17 & $<0.001$ & \\
\hline Main: distractor & & 25.90 & & 3,51 & $<0.001$ & \\
\hline Interaction & & 11.41 & & 3,51 & $<0.001$ & \\
\hline \multicolumn{7}{|l|}{ Effect } \\
\hline Control: lexical & 80 & & 8.72 & 12 & $<0.001$ & 60,100 \\
\hline Control: semantic & 46 & & 3.49 & 12 & 0.004 & 17,74 \\
\hline Control: phonological & 18 & & -1.77 & 12 & 0.102 & $-40,4$ \\
\hline Patient: lexical & 332 & & 5.21 & 5 & 0.003 & 168,495 \\
\hline Patient: semantic & 75 & & 0.81 & 5 & 0.452 & $-161,311$ \\
\hline Patient: phonological & 172 & & -3.07 & 5 & 0.028 & $-315,-28$ \\
\hline \multicolumn{7}{|c|}{ Distractor: patients vs. controls } \\
\hline Lexical & 251 & & 3.91 & 5.2 & 0.010 & 88,414 \\
\hline Semantic & 29 & & 0.31 & 5.2 & 0.766 & $-265,206$ \\
\hline Phonological & 172 & & 2.70 & 5.3 & 0.040 & 10,297 \\
\hline
\end{tabular}

Note: diff, difference.

lateral PFC. This finding is in line with the neuropsychological literature suggesting a critical role of the left lateral PFC (and the ventrolateral PFC in particular) for interference control in verbal tasks (Tsuchida and Fellows, 2013; Geddes et al., 2014)

The semantic interference effect in the patients was highly variable. Descriptively, three patients had longer RTs for the related than the unrelated condition (i.e., semantic interference, patients 3,4 , and 5 , with only one patient showing interference $2 S D$ s above the mean of the controls) and three patients showed the opposite effect (i.e., semantic facilitation, patients 1, 2, and 6, with one patient showing facilitation 2 SDs below the mean of the controls).

Facilitation from semantically related distractors is not a common finding in the literature. Wilshire et al. (2007) reported a case study of an anomic patient showing semantic facilitation with simultaneous presentation of picture and

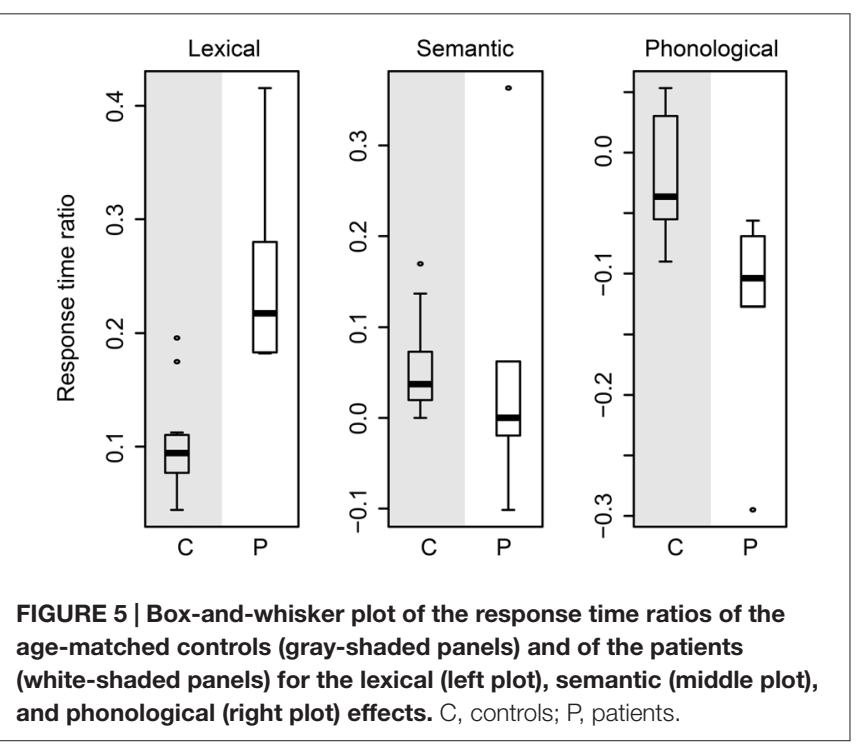

distractor (i.e., 0 ms stimulus onset asynchrony). However, given that all our six patients were not aphasic, comparisons with Wilshire et al.'s patient remain difficult. Other studies reporting semantic facilitation in picture-word interference had additional manipulations such as brief distractor pre-exposure, distractor masking, or low co-activation of distractors, which decreased the strength of the distractor as a competitor (e.g., Roelofs, 1992, 1993; Piai et al., 2012). It could be the case that, for the patients experiencing semantic facilitation, the dynamics of processing the distractor vs. the picture is altered for some reason, and the distractor is able to prime the picture name but not compete with it (for further discussion, see Piai et al., 2012). Additionally, it could be that the amount of ventrolateral PFC damage or the specific portion of the ventrolateral PFC damaged are critical in determining if and how much control processes will be impaired in the presence of semantic competitors. Rather than being a functionally uniform region, it has been argued that different portions of the ventrolateral PFC subserve different cognitive functions (Clos et al., 2013). Also, it could be the case that a larger lesion is more likely to encompass portions of the ventrolateral PFC that are critical to resolving competition among semantic alternatives. Variations in the extent of ventrolateral PFC affected could potentially explain the variable behavior of our patients regarding semantic effects. However, assessing these hypotheses directly is not possible in the present study. In Figure 6, we show the lesion overlap of the three patients showing facilitation vs. the three patients showing interference, as well as the overlap in lesion location between the two groups. As can be seen, although the three patients showing semantic interference have lesions maximally overlapping in the ventrolateral PFC, the group showing facilitation does not have a converging lesion maximum. Moreover, there seems to be great overlap in lesion location between the two groups. Thus, the extent to which left lateral PFC lesions affect the semantic interference effect in picture-word interference remains unclear at this point, but will hopefully be clarified in future investigations. 
Number of patients with lesion

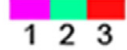

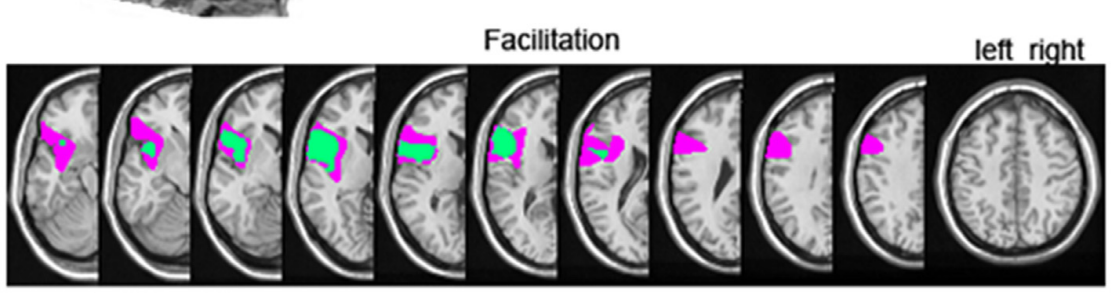

Interference
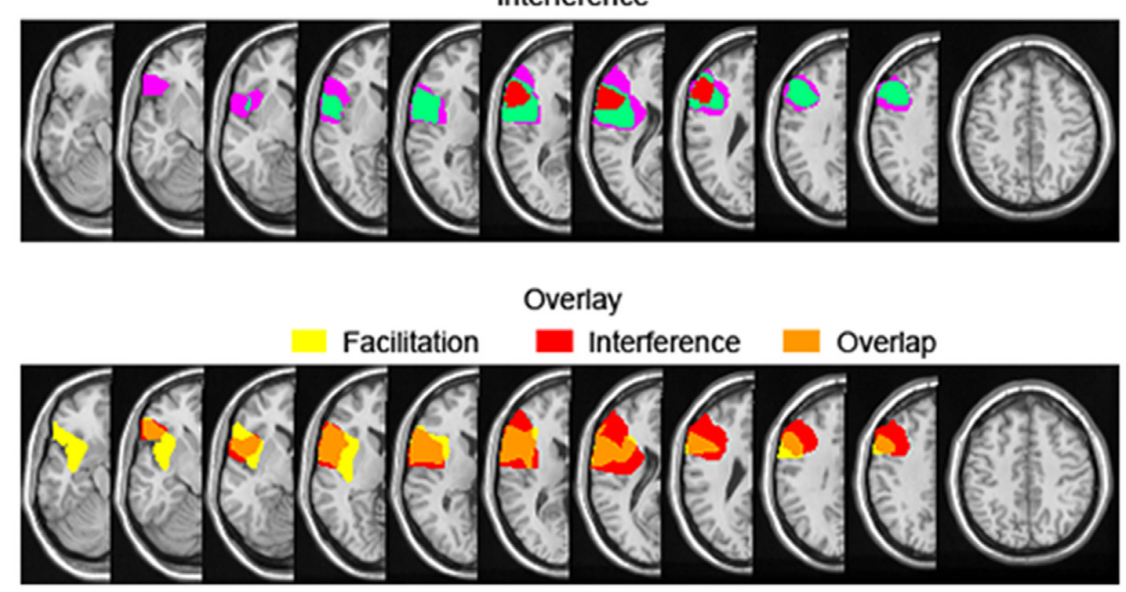

FIGURE 6 | Lesion overlap map of the three left prefrontal cortex patients showing descriptive semantic facilitation (upper) and semantic interference (middle), as well the lesion overlap between the semantic facilitation and interference groups (bottom). The color scale in the upper and middle panels indicates the number of patients with a lesion in a particular location. For the bottom overlay, lesion overlap of the facilitation group is given in yellow, for the interference group in red, and their overlap is shown in orange.

Using Bayesian estimation, we established that a zero difference in semantic interference in the patient group, on the group level, is a credible value given the data ${ }^{2}$. Thus, we can conclude that our patient group was not consistently impaired by semantic competitors. However, all six patients showed an increased lexical interference effect relative to controls. The dissociation between lexical and semantic interference in the patients' behavior may indicate different types of control functions necessary for resolving lexical vs. semantic interference, which are differentially dependent on the lateral PFC. In the introduction, we related performance in pictureword interference to a control function enabling participants to suppress distractors that might slow down picture naming. However, the reason why distractors may hinder picture naming can be diverse. For example, in the picture-word

\footnotetext{
${ }^{2}$ We note that accepting the hypothesis of zero difference between conditions is only possible when there is high enough precision in the estimation of the parameters. In that case, one defines an interval of parameter values that are negligibly different from the null value for practical purposes (e.g., an effect of $10 \mathrm{~ms}$ may be deemed negligibly different from $0 \mathrm{~ms}$ ). When all the credible parameter values fall within this interval, the null value is accepted. Unfortunately, our sample size is not large enough to yield very precise parameter estimations.
}

interference literature, distractor effects exist that are caused by the distractor words themselves, independent of their semantic relation to the picture. The distractor-frequency effect is such an effect, in which pictures paired with low-frequency distractors are named more slowly than with high-frequency distractors (Miozzo and Caramazza, 2003). It has been shown that the semantic interference and distractor-frequency effects reflect different underlying operations (Scaltritti et al., 2015; for a computational model formalizing the parameters associated with these effects, see also Roelofs, 2003). The semantic interference effect is thought to emerge due to competition between the picture name and the distractor word during lexical selection (e.g., Roelofs, 1992; La Heij et al., 2006; Rahman and Aristei, 2010). By contrast, the distractor-frequency effect is explained by a reactive blocking mechanism that blocks the distractor word so that processing of the target picture is favored (Roelofs, 2003). The speed with which the distractor can be processed determines the speed of blocking (e.g., Roelofs et al., 2011). The blocking mechanism is an early attentional mechanism that operates independently from resolving semantic competition (e.g., Roelofs, 2003).

The lexical interference effect conceivably measures the engagement of this early attentional, blocking mechanism. 
The mechanism is always necessary in conditions with a distractor word, such as the unrelated condition, so that processing the picture is favored over distractor processing. In the neutral condition, no blocking is necessary because no distractor word is presented. So the lexical interference effect maximally captures the workings of the blocking mechanism. The semantic interference effect is not informative about the involvement of early attentional blocking demands, because the semantic and unrelated conditions both require blocking of the distractor word.

In our study, resolving lexical interference was impaired in all six left PFC patients whereas resolving semantic interference was not. This dissociation implies that the left PFC is critically involved in attentional distractor blocking so that picture processing is prioritized despite distracting information. But the left PFC is not necessarily required for resolving semantic competition between picture name and distractor word during response selection. This latter proposal is in line with recent evidence that the left lateral PFC is not always necessary to resolve interference from semantically related competitors (Riès et al., 2015). In this recent study comparing the performance of left PFC patients in two production tasks inducing semantic interference, Riès et al. (2015) found that left PFC patients had a larger semantic interference effect than controls in blockedcyclic naming, a picture-naming task that requires proactive interference control due to blocked design and picture repetition. By contrast, in a continuous picture naming task in which pictures are not repeated (Howard et al., 2006), the same patients and controls had an interference effect of similar magnitude.

An additional finding of our study was that the phonological facilitation effect was larger in the patients than in the control participants, similar to the findings of Hashimoto and Thompson (2010) in their aphasic patients. It is well known that patients with word finding difficulties benefit from phonemic cues (e.g., Li and Williams, 1989). Although our patients performed within normal limits on the WAB and thus, were not aphasic as the patients in Hashimoto and Thompson (2010), it is common for this patient group (i.e., within normal limits-WAB) to still have subclinical language impairment beyond what would be expected for their age (e.g., Riès et al., 2015). In fact, such a suggestion finds support in our own data. As reported above, patients were overall slower than the controls. Perhaps even more importantly, in the XXX condition, in which the distracting information is neutral, patients were still slower $(250 \mathrm{~ms}$ on average) than the controls. This finding is consistent with residual word-finding difficulties these patients often report.

\section{REFERENCES}

Alario, F.-X., Chainay, H., Lehericy, S., and Cohen, L. (2006). The role of the supplementary motor area (SMA) in word production. Brain Res. 1076, 129-143. doi: 10.1016/j.brainres.2005. 11.104

Alvarez, J. A., and Emory, E. (2006). Executive function and the frontal lobes: a meta-analytic review. Neuropsychol. Rev. 16, 17-42. doi: 10.1007/s11065-0069002-x
It is thus possible to explain the hyper phonological facilitation in these patients in that they might have developed a greater reliance on phonemic cues in comparison to controls when having wordfinding problems. However, this account remains speculative at this point. Crucially, all six patients showed an increased lexical interference effect, providing evidence that any damage to the left lateral PFC will affect the ability to carry out picture naming in the presence of competing linguistic information.

\section{CONCLUSION}

In conclusion, all six left PFC patients showed an increased lexical interference effect. The impaired performance of the patients in naming pictures in the presence of a competing distractor word (i.e., linguistic competing information) provides evidence that the left lateral PFC is a necessary structure in providing control over word production processes. This finding corroborates the evidence that word production requires attentional control and further specifies that interference control in word production necessarily implicates the lateral PFC.

\section{AUTHOR CONTRIBUTIONS}

Conceptualized and designed the experiment (DS); acquired the data (DS); analyzed the data (VP); wrote the article (VP, SKR, DS). All authors have approved the final version of the article and agree to be accountable for all aspects of this work.

\section{ACKNOWLEDGMENTS}

We would like to thank the patients for participating in this study. We are grateful to Deborah M. Burke, Mathieu Koppen, Robert T. Knight, Clay Clayworth, Brian Curran, Nina F. Dronkers, Juliana Baldo, Caitlin Roxby, Ardi Roelofs, and the Aphasia and Cognitive Neuroscience Labs at the VA for their contributions on various aspects of this work. The authors are funded by grants from the Netherlands Organization for Scientific Research (VP, grant number 446-13-009), the National Institute on Deafness and Other Communication Disorders (SKR, grant number F32DC013245 and DS, grant number DC03424), the National Science Foundation (DS, grant number 0236818), and the Department of Veterans Affairs (DS, grant number CX000566). The content is solely the responsibility of the authors and does not necessarily represent the official views of the National Institutes of Health, the Department of Veterans Affairs, or the United States Government. 
co-activation-based parcellation of area 44. Neuroimage 83, 174-188. doi: 10. 1016/j.neuroimage.2013.06.041

Cramer, A. O. J., van Ravenzwaaij, D., Matzke, D., Steingroever, H., Wetzels, R., Grasman, R. P. P. P., et al. (2015). Hidden multiplicity in multiway ANOVA: prevalence, consequences and remedies. Psychon. Bull. Rev. doi: 10. 3758/s13423-015-0913-5 [Epub ahead of print].

Damian, M., and Martin, R. (1999). Semantic and phonological codes interact in single word production. J. Exp. Psychol. Learn. Mem. Cogn. 25, 345-361. doi: 10. 1037/0278-7393.25.2.345

de Zubicaray, G. I., Hansen, S., and McMahon, K. L. (2013). Differential processing of thematic and categorical conceptual relations in spoken word production. J. Exp. Psychol. Gen. 142, 131-142. doi: 10.1037/a0028717

de Zubicaray, G. I., and McMahon, K. L. (2009). Auditory context effects in picture naming investigated with event-related fMRI. Cogn. Affect. Behav. Neurosci. 9, 260-269. doi: 10.3758/CABN.9.3.260

de Zubicaray, G. I., Wilson, S. J., McMahon, K. L., and Muthiah, S. (2001). The semantic interference effect in the picture-word paradigm: an event-related fMRI study employing overt responses. Hum. Brain Mapp. 14, 218-227. doi: 10. 1002/hbm.1054

Friedman, N. P., and Miyake, A. (2004). The relations among inhibition and interference control functions: a latent-variable analysis. J. Exp. Psychol. Gen. 133, 101-135. doi: 10.1037/0096-3445.133.1.101

Geddes, M. R., Tsuchida, A., Ashley, V., Swick, D., and Fellows, L. K. (2014). Material-specific interference control is dissociable and lateralized in human prefrontal cortex. Neuropsychologia 64, 310-319. doi: 10.1016/j. neuropsychologia.2014.09.024

Geranmayeh, F., Wise, R. J. S., Mehta, A., and Leech, R. (2014). Overlapping networks engaged during spoken language production and its cognitive control. J. Neurosci. 34, 8728-8740. doi: 10.1523/JNEUROSCI.0428-14.2014

Glaser, W. R., and Düngelhoff, F. J. (1984). The time course of picture-word interference. J. Exp. Psychol. Hum. Percept. Perform. 10, 640-654. doi: 10. 1037/0096-1523.10.5.640

Hashimoto, N., and Thompson, C. K. (2010). The use of the picture-word interference paradigm to examine naming abilities in aphasic individuals. Aphasiology 24, 580-611. doi: 10.1080/02687030902777567

Howard, D., Nickels, L., Coltheart, M., and Cole-Virtue, J. (2006). Cumulative semantic inhibition in picture naming: experimental and computational studies. Cognition 100, 464-482. doi: 10.1016/j.cognition.2005.02.006

Indefrey, P. (2011). The spatial and temporal signatures of word production components: a critical update. Front. Psychol. 2:255. doi: 10.3389/fpsyg.2011. 00255

Kertesz, A. (1982). Western Aphasia Battery Test Manual. New York: Grune and Stratton.

Kruschke, J. K. (2013). Bayesian estimation supersedes the $t$ test. J. Exp. Psychol. Gen. 142, 573-603. doi: 10.1037/a0029146

Kruschke, J. K. (2015). Doing Bayesian Data Analysis: A Tutorial with R, JAGS, and Stan, 2nd Edn. (Waltham, MA: Academic Press/Elsevier).

La Heij, W., Kuipers, J. R., and Starreveld, P. A. (2006). In defense of the lexical-competition account of picture-word interference: a comment on Finkbeiner and Caramazza (2006). Cortex 42, 1028-1031. doi: 10.1016/s00109452(08)70209-0

Li, E. C., and Williams, S. E. (1989). The efficacy of two types of cues in aphasic patients. Aphasiology 3, 619-626. doi: 10.1080/026870389082 49028

Miozzo, M., and Caramazza, A. (2003). When more is less: a counterintuitive effect of distractor frequency in the picture-word interference paradigm. J. Exp. Psychol. Gen. 132, 228-252. doi: 10.1037/0096-3445.132.2.228

Nigg, J. T. (2000). On inhibition/disinhibition in developmental psychopathology: views from cognitive and personality psychology and a working inhibition taxonomy. Psychol. Bull. 126, 220-246. doi: 10.1037/0033-2909. 126.2.220

Petrides, M. (2005). Lateral prefrontal cortex: architectonic and functional organization. Philos. Trans. R Soc. Lond. B Biol. Sci. 360, 781-795. doi: 10. 1098/rstb.2005.1631

Piai, V., Roelofs, A., Acheson, D. J., and Takashima, A. (2013). Attention for speaking: domain-general control from the anterior cingulate cortex in spoken word production. Front. Hum. Neurosci. 7:832. doi: 10.3389/fnhum.2013. 00832
Piai, V., Roelofs, A., and Schriefers, H. (2012). Distractor strength and selective attention in picture-naming performance. Mem. Cognit. 40, 614-627. doi: 10. 3758/s13421-011-0171-3

Plummer, M. (2014). rjags: Bayesian Graphical Models using MCMC. (R package version 3-14). Available online at: http://CRAN.R-project.org/package=rjags

Posner, M. I., and Petersen, S. E. (1990). The attention system of the human brain. Annu. Rev. Neurosci. 13, 25-42. doi: 10.1146/annurev.neuro.13.1.25

Price, C. J. (2012). A review and synthesis of the first 20 years of PET and fMRI studies of heard speech, spoken language and reading. Neuroimage 62, 816-847. doi: 10.1016/j.neuroimage.2012.04.062

Rahman, R. A., and Aristei, S. (2010). Now you see it ... and now again: semantic interference reflects lexical competition in speech production with and without articulation. Psychon. Bull. Rev. 17, 657-661. doi: 10.3758/PBR. 17.5.657

R Development Core Team. (2014). R: A Language and Environment for Statistical Computing. R Foundation for Statistical Computing, Vienna. Available online at: http://www.R-project.org/

Riès, S. K., Greenhouse, I., Dronkers, N. F., Haaland, K. Y., and Knight, R. T. (2014). Double dissociation of the roles of the left and right prefrontal cortices in anticipatory regulation of action. Neuropsychologia 63, 215-225. doi: 10 1016/j.neuropsychologia.2014.08.026

Riès, S. K., Karzmark, C., Navarrete, E., Dronkers, N., and Knight, R. T. (2015). Specifying the role of the left prefrontal cortex in word selection. Brain Lang. 149, 135-147. doi: 10.1016/j.bandl.2015.07.007

Roelofs, A. (1992). A spreading-activation theory of lemma retrieval in speaking. Cognition 42, 107-142. doi: 10.1016/0010-0277(92)90041-f

Roelofs, A. (1993). Testing a non-decompositional theory of lemma retrieval in speaking: retrieval of verbs. Cognition 47, 59-87. doi: 10.1016/00100277(93)90062-Z

Roelofs, A. (2003). Goal-referenced selection of verbal action: modeling attentional control in the stroop task. Psychol. Rev. 110, 88-125. doi: 10.1037/0033-295x. 110.1 .88

Roelofs, A., and Piai, V. (2011). Attention demands of spoken word planning: a review. Front. Psychol. 2:307. doi: 10.3389/fpsyg.2011.00307

Roelofs, A., Piai, V., and Schriefers, H. (2011). Selective attention and distractor frequency in naming performance: comment on Dhooge and Hartsuiker (2010). J. Exp. Psychol. Learn. Mem. 37, 1032-1038. doi: 10.1037/a00 23328

Rosinski, R. R., Golinkoff, R. M., and Kukish, K. S. (1975). Automatic semantic processing in a picture-word interference task. Child Dev. 46, 247-253. doi: 10. 2307/1128859

Scaltritti, M., Navarrete, E., and Peressotti, F. (2015). Distributional analyses in the picture-word interference paradigm: exploring the semantic interference and the distractor frequency effects. Q. J. Exp. Psychol. (Hove) 68, 1348-1369. doi: 10.1080/17470218.2014.981196

Schnur, T. T., Schwartz, M. F., Brecher, A., and Hodgson, C. (2006). Semantic interference during blocked-cyclic naming: evidence from aphasia. J. Mem. Lang. 54, 199-227. doi: 10.1016/j.jml.2005.10.002

Schriefers, H., Meyer, A. S., and Levelt, W. J. M. (1990). Exploring the time course of lexical access in language production: picture-word interference studies. J. Mem. Lang. 29, 86-102. doi: 10.1016/0749-596x(90)90011-n

Shao, Z., Roelofs, A., Martin, R. C., and Meyer, A. S. (2015). Selective inhibition and naming performance in semantic blocking, picture-word interference and color-word stroop tasks. J. Exp. Psychol. Learn. Mem. Cogn. 41, 1806-1820. doi: $10.1037 / \mathrm{a} 0039363$

Snodgrass, J. G., and Vanderwart, M. (1980). A standardized set of 260 pictures: norms for name agreement, image agreement, familiarity and visual complexity. J. Exp. Psychol. Hum. Learn. 6, 174-215. doi: 10.1037/0278-7393.6. 2.174

Szczepanski, S. M., and Knight, R. T. (2014). Insights into human behavior from lesions to the prefrontal cortex. Neuron 83, 1002-1018. doi: 10.1016/j.neuron. 2014.08.011

Taylor, J. K., and Burke, D. M. (2002). Asymmetric aging effects on semantic and phonological processes: naming in the picture-word interference task. Psychol. Aging 17, 662-676. doi: 10.1037/0882-7974.17.4.662

Tsuchida, A., and Fellows, L. K. (2013). Are core component processes of executive function dissociable within the frontal lobes? Evidence from humans with focal prefrontal damage. Cortex 49, 1790-1800. doi: 10.1016/j.cortex.2012.10.014 
Unsworth, N. (2010). Interference control, working memory capacity and cognitive abilities: a latent variable analysis. Intelligence 38, 255-267. doi: 10. 1016/j.intell.2009.12.003

Wilshire, C. E., Keall, L. M., Stuart, E. J., and O’Donnell, D. J. (2007). Exploring the dynamics of aphasic word production using the picture-word interference task: a case study. Neuropsychologia 45, 939-953. doi: 10.1016/j.neuropsychologia. 2006.08.026

Yuan, P., and Raz, N. (2014). Prefrontal cortex and executive functions in healthy adults: a meta-analysis of structural neuroimaging studies. Neurosci. Biobehav. Rev. 42, 180-192. doi: 10.1016/j.neubiorev.2014.02.005
Conflict of Interest Statement: The authors declare that the research was conducted in the absence of any commercial or financial relationships that could be construed as a potential conflict of interest.

Copyright (C) 2016 Piai, Riès and Swick. This is an open-access article distributed under the terms of the Creative Commons Attribution License (CC BY). The use, distribution and reproduction in other forums is permitted, provided the original author(s) or licensor are credited and that the original publication in this journal is cited, in accordance with accepted academic practice. No use, distribution or reproduction is permitted which does not comply with these terms. 